\title{
Distribution and characterization of tumor-associated macrophages/microglia in rat $\mathrm{C6}$ glioma
}

\author{
ZHI-MING ZHANG $^{1 *}$, ZICHENG YANG ${ }^{2 *}$ and ZHIREN ZHANG ${ }^{2}$ \\ ${ }^{1}$ Department of Medicine, Shunde Polytechnic, Foshan, Guangdong 528300; ${ }^{2}$ Institute of Immunology, \\ Third Military Medical University of the People's Liberation Army, Chongqing 400038, P.R. China
}

Received September 4, 2014; Accepted June 22, 2015

DOI: $10.3892 / \mathrm{ol} .2015 .3533$

\begin{abstract}
Immunity responses and immunotherapy are novel areas of research for the pathological development and treatment of glioma, the most common brain cancer. Characterization of the subpopulations of infiltrated immune cells may aid in our understanding of the tumor immune response and contribute to the identification of cellular targets for selective immunotherapy. Using a rat C6 glioma model, the present study observed a significant heterogeneity of active macrophages and microglia, including cluster of differentiation $8(\mathrm{CD} 8)^{+}$, endothelial monocyte-activating polypeptide II $\left(\mathrm{EMAPII}^{+}\right.$and $\mathrm{ED}^{+}$cells, mostly in the areas of compact tumor growth and inside or around the pannecrosis. Moreover, the $\mathrm{CD}^{+}$cells were similar to reactive $\mathrm{ED}^{+}$and $\mathrm{EMAPII}^{+}$ microglia/macrophages in morphology and distribution, but different from the W3/13 ${ }^{+} \mathrm{T}$ cells. These observations suggest that different subtypes of macrophages and microglia are involved in glioma development and thus, may be potential targets for immunotherapeutic antitumor strategies.
\end{abstract}

\section{Introduction}

Glioma, which occurs in the central nervous system (CNS), is the most common primary malignant tumor, with an unacceptably poor prognosis despite the application of a variety of treatments, including surgery, radiotherapy and chemotherapy (1). In recent years, accumulated data have established that the immune network may be involved in regulating

Correspondence to: Miss Zhiren Zhang, Institute of Immunology, Third Military Medical University of the People's Liberation Army, 30 Gaotanyan Main Street, Chongqing 400038, P.R. China

E-mail: zhangzhiren@yahoo.com

${ }^{*}$ Contributed equally

Abbreviations: CNS, central nervous system; EMAPII, endothelial monocyte-activaing polypeptide II; CD8, cluster of differentiation 8

Key words: glioblastoma, microglia, macrophages, endothelial monocyte-activating polypeptide II, CD68, CD8 glioma development and growth (2). Moreover, tumor cells have been killed following direct immunization experiments using vaccines in mice, indicating its role in specific protective immunity and tumor destruction mediated by immune cells, such as microglia/macrophages (3). These immunotherapies exert a highly specific, long-term fatal effect on tumor cells by stimulating and supplementing the body's antitumor immunity $(4,5)$, with only minimal adverse reactions (6). However, in the past decade, the main debate has focused on whether immunity exhibits an antitumor role in the CNS or functions as a tumor growth promoter (7). Additionally, the functions and mechanism of different immune cells in glioma require further investigation.

Various pathological stimuli, including brain injury (8), neurodegeneration (9), infection, inflammation (10) and brain tumors (11), can activate brain macrophages/microglia. It has been observed that in CNS tumors, activated microglia/macrophages can engulf tissue debris and secrete growth-promoting factors, leading to regeneration (12). By contrast, the overactivation of microglia/macrophages will lead to tissue damage in the CNS (13). Following activation, microglia/macrophages change their morphology, upregulate the expression of certain membrane proteins and produce certain cytokines, resulting in inflammation and tissue loss (14). The contrast in roles played by activated microglia/macrophages may be due to the existence of different subpopulations of microglia/macrophages (15).

Endothelial monocyte-activating polypeptide II (EMAPII) ${ }^{+}$ macrophages secrete proinflammatory and antiangiogenic cytokine in the parenchyma of the CNS, stimulate the accumulation and cytokine production of macrophages, and trigger the apoptosis of endothelial cells (16). Increased EMAPII expression is considered to be a sensitive marker of neurotoxic lesions in rat brains (17). ED1, which is mainly found in phagocytosing macrophages and reactive microglia, is a lysosomal membrane protein. Macrophages with ED1 can be observed in the majority of brain tumors, as well as in gliomas (18). Cluster of differentiation 8 (CD8) was traditionally recognized as a marker that is mainly expressed in cytotoxic $\mathrm{T}$ cells and natural killer cells (19). More recently, studies have reported the accumulation of $\mathrm{CD}^{+}$macrophages in various $\mathrm{CNS}$ pathologies, such as ischemia, Wallerian degeneration and spinal cord injury $(19,20)$. CD8 $8^{+}$cells, no matter whether lymphocytes or macrophages, may have important distinctive 
functions in glioma. However, information on the role played by different subsets of macrophages/microglia in glioma is relatively lacking.

Further understanding of immune-tumor interactions will benefit tumor therapies. Moreover, it has been demonstrated that microglial cells are found in necrotic and intact areas of brain tumor tissues (21). Thus, in the present study, multiple antigens were used to study the distribution and characteristics of tumor-associated macrophages/microglia in rat C6 glioma.

\section{Materials and methods}

Rat brain tissue libraries. The C6 glioma cell-induced rat brain tissue was a gift from Professor Hermann J. Schluesener (Institute of Brain Research, Tübingen University, Tübingen, Germany). The C6 glioma cells were injected into the brains of Sprague-Dawley rats, as previously reported (22).

Immunohistochemistry. Brain sections were dewaxed and then boiled for $15 \mathrm{~min}$ in citrate buffer $(2.1 \mathrm{~g}$ sodium citrate/1; $\mathrm{pH} 6$ ) in a $600-\mathrm{W}$ microwave oven. $1 \% \mathrm{H}_{2} \mathrm{O}_{2}$ in pure methanol was used to inhibit endogenous peroxidase activity for $15 \mathrm{~min}$. Following blocking with $10 \%$ normal pig serum (Biochrom, Berlin, Germany) for $15 \mathrm{~min}$, the rat brain sections $(0.5 \mu \mathrm{m})$ were incubated with the following mouse monoclonal antibodies overnight at $4^{\circ} \mathrm{C}$ : OX8 (1:100; AbD Serotec, Oxford, UK), ED1 (1:100; AbD Serotec), EMAPII (1:100; BMA Biomedicals, Augst, Switzerland), W3/13 (1:100; AbD Serotec), OX6 (1:100; AbD Serotec), OX62 (1:50; AbD Serotec) or OX22 (1:100; AbD Serotec). Subsequently, the sections were incubated with biotinylated swine anti-rabbit (DAKO, Hamburg, Germany) immunoglobulin (Ig)G F(ab)2 antibody fragment or a biotinylated rabbit anti-mouse IgG F(ab)2 antibody fragment (Dako, Hamburg, Germany). Following washing with phosphate-buffered saline with Tween 20 (Beijing SHX Biotechnology Co., Ltd., Beijing, China) and further incubation with a Streptavidin-Avidin-Biotin complex (Dako) for $30 \mathrm{~min}$ at room temperature, the sections were developed with diaminobenzidine substrate (Fluka, Neu-Ulm, Germany) for visualization. Finally, the tissue sections were counterstained with hematoxylin. With regard to the negative controls, the primary antibodies were omitted. Hematoxylin and eosin staining was additionally applied to evaluate the lesions of the brain trauma sections.

Light microscopic examination of immunostained brain sections. The immunostaining was examined and the numbers of positive cells at the brain tumor areas were quantified. For each section, the numbers of $\mathrm{OX}^{+}, \mathrm{ED}^{+}$, $\mathrm{EMAPII}^{+}$or $\mathrm{W} 3 / 13^{+}$ cells were counted in 5 non-overlapping high-power fields (HPF; x400 magnification). Areas around the pannecrotic border that had a maximum number of positive cells were used as the selected HPF. Only positive cells with the nucleus at the focal plane were counted, while positive perivascular cells were not counted in each field studied. Counting was performed by individuals who were blinded to the treatment. The results of the numbers of positive cells per HPF are presented as the arithmetic mean \pm standard error of the mean.
Statistical analysis. Graph Pad Prism 4.0 (GraphPad Software, Inc., La Jolla, CA, USA) was used for the statistical analysis. In-group and between-group differences were studied by one-way analysis of variance followed by Dunnett's multiple comparisons test. $\mathrm{P}<0.05$ was used to indicate a statistically significant difference.

\section{Results}

Activated microglia/macrophage accumulation in brains of the rat C6 glioma model. The infiltration of reactive microglia/macrophages was analyzed in rat C6 gliomas in this study by immunohistochemistry with the antibodies ED1 and EMAPII. The ED1 antibody stains CD68, which is mainly found in phagocytosing macrophages and reactive microglia (23). The upregulated expression of CD68 usually indicates an increase in phagocytical activity of the marked cells. In the C6 gliomas, little infiltration of $\mathrm{ED}^{+}$cells was observed in the areas of infiltrated tumor growth (Fig. 1A). Slight accumulation of ED1 ${ }^{+}$cells was observed in the border areas (Fig. 1A), while relatively strong infiltration was detected inside the areas of pannecrosis (Fig. 1B) and in the areas of compact tumor growth (Fig. 1A and C).

EMAPII, which is expressed by activated microglial/macrophages in the CNS parenchyma, is a proinflammatory cytokine (16). The expression of EMAPII is considered a sensitive marker of microglia activation. In the present study, similar to ED1 staining, an accumulation of $\mathrm{EMAPII}^{+}$cells was observed in the C6 tumor areas (Fig. 1D and F). However, the number of $\mathrm{EMAPII}^{+}$cells was greater than that of the ED1 ${ }^{+}$ cells, and the EMAPII ${ }^{+}$cells were located more at the borders of the pannecrosis rather than inside (Fig. 1E).

Single immunohistochemical staining of CD8 in brains of the rat C6 glioma model. Next, CD8 expression was detected in the rat C6 glioma brains by immunohistochemistry. Little CD8 expression was observed in the areas of infiltrating tumor growth (Fig. 2A). Slight infiltration of $\mathrm{CD}^{+}$cells was observed in the border areas (Fig. 2A), while a relatively strong accumulation was detected in areas of compact tumor growth (Fig. 2A and C). Moreover, the accumulation of $\mathrm{CD}^{+}$cells in perivasculature areas was observed (Fig. 2D). Furthermore, a strong infiltration of $\mathrm{CD}^{+}$cells was observed in areas of compact tumor growth and at the edge of the pannecrosis (Fig. 2B). However, inside the pannecrosis areas (Fig. 2B) and in normal neuronal areas (Fig. 2E), much fewer $\mathrm{CD}^{+}$cells were observed. Morphologically, the majority of the accumulated $\mathrm{CD}^{+}$cells were similar to macrophage phenotypes, with amoeboid morphological characteristics (Fig. 2C).

ED1 and EMAPII label activated microglia/macrophages, and in the present study, the time course of accumulation, the morphology and the distribution of $\mathrm{CD}^{+}$cells were similar to those of the $\mathrm{ED}^{+}$and $\mathrm{EMAPII}^{+}$cells. Similar to the majority of $\mathrm{ED}^{+}$and $\mathrm{EMAPII}^{+}$cells, the $\mathrm{CD}^{+}$cells showed amoeboid morphological characteristics in the pannecrosis (Figs. 1C, 1F, 2C and 2D). Moreover, the time course of $\mathrm{CD}^{+}$ cell infiltration in the tumor areas was parallel to that of the $\mathrm{ED}^{+}$and $\mathrm{EMAPII}^{+}$cells. In addition, the $\mathrm{EDI}^{+}$and $\mathrm{EMAPII}^{+}$ cells were located inside (Fig. 2B) and at the border (Fig. 2E) of pannecrosis areas, which was similar for the $\mathrm{CD} 8^{+}$cells (Fig. 2B-D). 

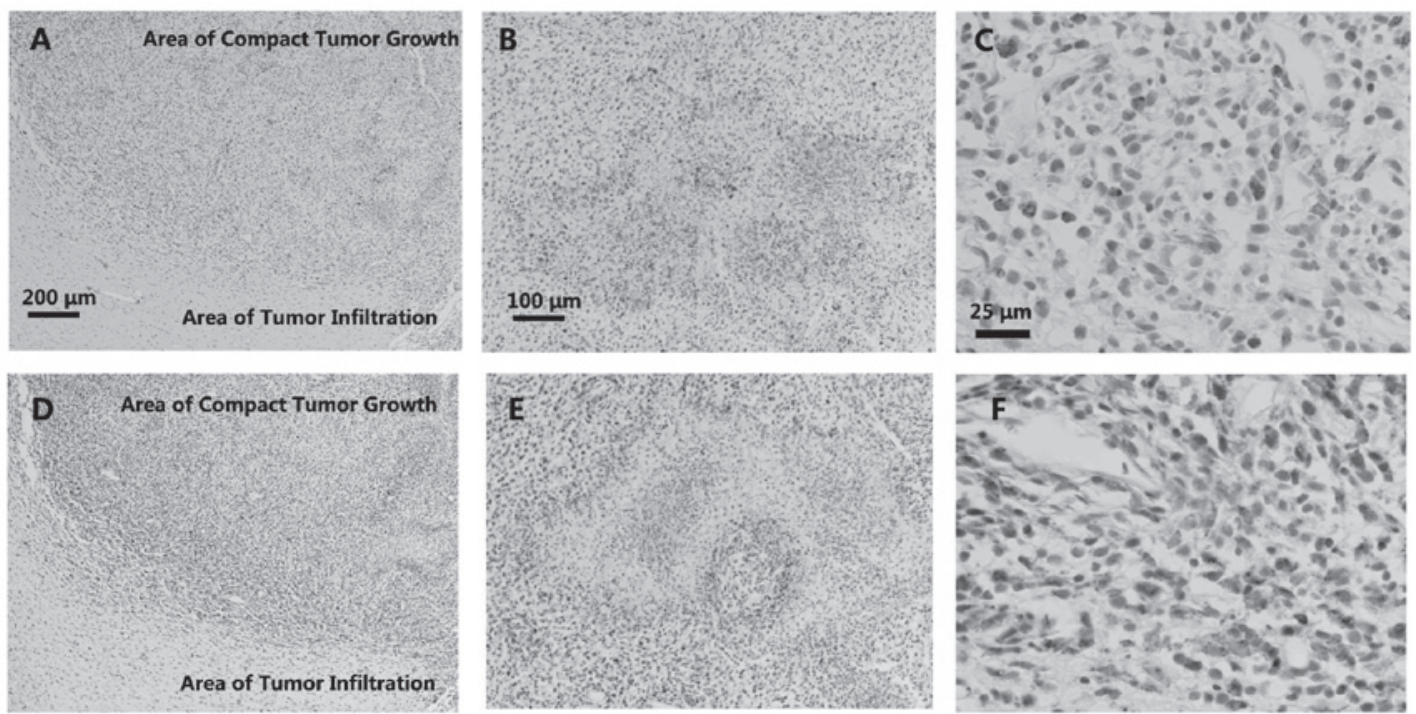

Figure 1. Immunohistochemical labelling of ED1 and endothelial monocyte-activating polypeptide II (EMAPII) in the areas of (A and D) the tumor border, (B and E) pannecrosis and ( $\mathrm{C}$ and F) compact tumor growth. (A) ED1 ${ }^{+}$and (D) EMAPII cells were detected in the areas of compact tumor growth and seldomly observed in the areas of tumor infiltration; (A) and (D) show both compact glioma and tumor infiltration areas. (B) Several ED1 ${ }^{+}$cells were also observed in the pannecrosis, (C) just like the relative strong recruitment of $\mathrm{EDI}^{+}$cells in areas of the compact tumor growth, while EMAPII ${ }^{+}$cells were observed in (F) the areas of compact tumor growth and (E) only in the borders of the pannecrosis.
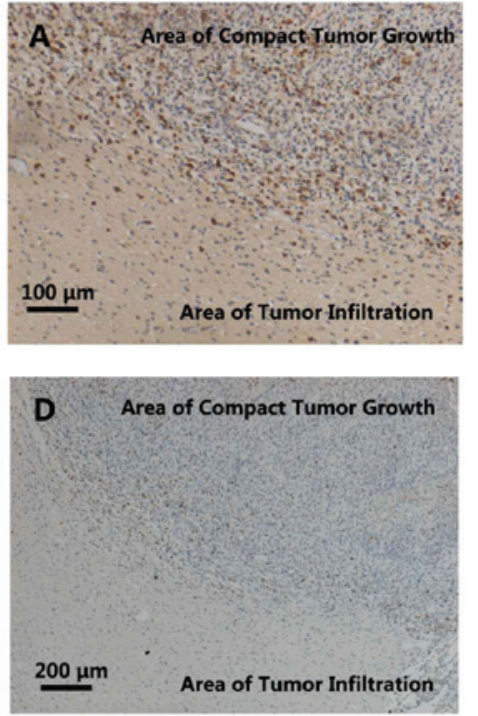
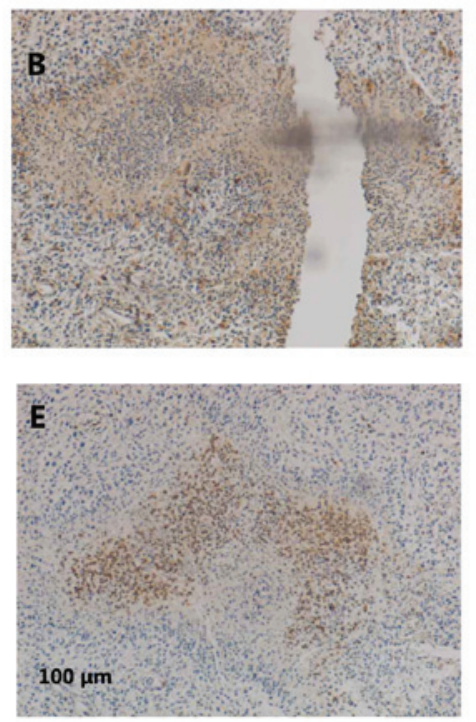
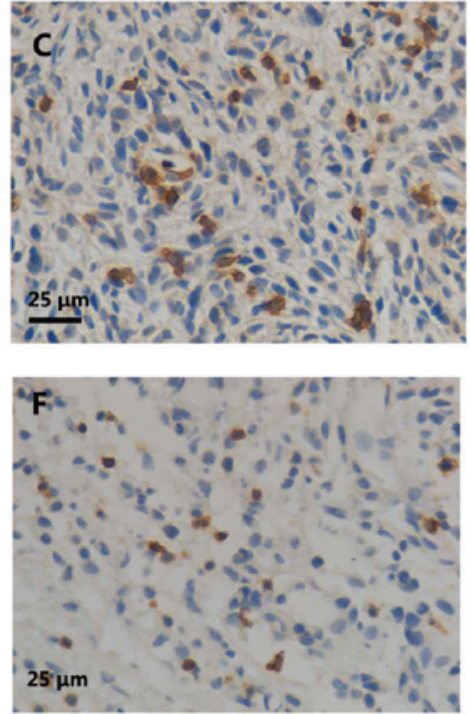

Figure 2. Immunohistochemical labelling of OX8 and W3/13 in (A and D) the areas of the tumor border, (B and E) pannecrosis and (C and F) compact tumor growth, in the rat $\mathrm{C} 6$ glioma brains. Immunostaining of brain coronal section showed a decreased density of OX8 ${ }^{+}$cells in areas of tumor infiltration compared with areas of compact tumor growth; (A) and (D) show both compact glioma and tumor infiltration areas. (B) In the border of the pannecrosis, a strong accumulation of $\mathrm{OX}^{+}$cells was observed. (C) The $\mathrm{OX} 8^{+}$cells were mostly located in the areas of tumor growth. However, the infiltration of several W3/13 cells could be detected (D) in the borders and (F) a few in areas of compact tumor growth. (E) The cells were widely observed in the areas of the pannecrosis.

T cells accumulation in the brains of the rat C6 glioma model. Since $\mathrm{CD}^{+}$is traditionally observed in $\mathrm{T}$ cells, the present study further detected $\mathrm{T}$ cell accumulation in the rat $\mathrm{C} 6$ gliomas using a pan-T-cell marker, W3/13. In contrast to the $\mathrm{CD}^{+}$cells (Figs. 1A, 1D and 2A), the infiltration of the W3/13 ${ }^{+}$cells was only seldomly observed in the borders of the compact tumor growth areas (Fig. 2A). Furthermore, the $\mathrm{W} 3 / 13^{+}$cells exhibited smaller cell bodies (Fig. 2C) compared with the $\mathrm{CD}^{+}$cells (Fig. 2C). Therefore, the time course of accumulation, morphology and distribution all suggested that the major cellular source of CD8 was reactive macrophages/microglia, but not $\mathrm{T}$ cells.

\section{Discussion}

In the present study, the early accumulation, distribution and characteristics of $\mathrm{CD}^{+}, \mathrm{ED}^{+}, \mathrm{EMAPII}^{+}$and $\mathrm{W} 3 / 13^{+}$cells in rat C6 glioma brains were analyzed. Significant ED1 ${ }^{+}$cell accumulation was observed mostly located to areas of compact tumor growth and notably distributed inside the pannecrosis. EMAPII $^{+}$cell accumulation was also detected mainly in the areas of compact tumor growth, but only at the borders of the pannecrosis. It is noteworthy that the morphology and distribution of the $\mathrm{CD}^{+}$cells were similar to those of the reactive 
$\mathrm{ED}^{+}$and $\mathrm{EMAPII}^{+}$macrophages and microglia. However, the morphology and localization of the $\mathrm{CD} 8^{+}$cells were different from those of the few infiltrating $\mathrm{W} 3 / 13^{+} \mathrm{T}$ cells These results were expected as they were the same as those found previously (24). Thus, this observation means that it is reasonable to speculate that the $\mathrm{CD}^{+}$cells were also $\mathrm{ED}^{+}$and EMAPII $^{+}$macrophages/microglia. The heterogeneity of activated macrophages and microglia in rat C6 gliomas represent diverse subpopulations with different but relative functions, which may play a crucial role in the growth of glioma.

EMAPII is a well-known proinflammatory and antiangiogenic cytokine, which is expressed by activated macrophages/microglia in the parenchyma of the CNS (16). The pathophysiological expression of EMAPII induces the infiltration and activation of macrophages and endothelial apoptosis (16). Increased EMAPII expression is regarded as a sensitive marker of neurotoxic lesions in the rat brain (17). ED1 is an indication of activated macrophages expressed in the majority of tumor-associated macrophages (25). The detection of these two subsets may indicate a positive role in the formation of necrosis.

CD8 has been classically viewed as a marker of a certain subpopulation of lymphocytes (19). More recently, the accumulation of $\mathrm{CD}^{+}$macrophages has been observed in several pathologies of the CNS, such as ischemia (26), Wallerian degeneration (27), experimental autoimmune encephalomyelitis (26), bornavirus encephalitis (28), spinal cord injury (20) and glomerulonephritis (29). In the present study, nearly all $\mathrm{CD}^{+}$cells shared a similar morphology and distribution with reactive $\mathrm{EDI}^{+}$and $\mathrm{EMAPII}^{+}$macrophages and/or microglia, but were not similar to the few infiltrating $\mathrm{W} 3 / 13^{+} \mathrm{T}$ cells This similar morphology and distribution of reactive $\mathrm{CD} 8^{+}$, $\mathrm{ED}^{+}$and $\mathrm{EMAPII}^{+}$cells suggests that the observed reactive microglia/macrophages were $\mathrm{CD}^{+}$. Moreover, the presumable $\mathrm{CD}^{+}$macrophages/microglia were mainly observed in areas of the compact tumor growth and concentrated at the boundary of the pannecrosis. The presumable $\mathrm{CD}^{+}$macrophages/microglia have been observed to be limited to regions of cavitation lesions following cerebral ischemia (26) and spinal cord injury (20). The restricted distribution arrangement of $\mathrm{CD}^{+}$phagocytes in rat $\mathrm{C} 6$ glioma indicates a crucial role in regulating responses in tumor necrosis.

The contributions of the $\mathrm{CD} 8^{+}$microglia/macrophages to the development of CNS damage are not fully understood. In alveolar macrophages, the ligation of CD8 molecules resulted in the production of the inducible nitric oxide synthase, nitric oxide, tumor necrosis factor (TNF)- $\alpha$ and interleukin-1 $\beta$, and enhanced the cytotoxicity to Leishmania major (30). Furthermore, the infiltration of $\mathrm{CD}^{+}$microglia/macrophages was mainly located to severe inflammatory destruction tissue in experimental autoimmune encephalomyelitis, suggesting their contribution to the pathological process (26).

In a more traditional view, OX8 is the marker of a subtype of activated T cells, which can also be observed in glioma (24). In the present study, the observation of $\mathrm{W} 3 / 13^{+}$cells confirmed the existence of $\mathrm{T}$ cells in glioma, which was in accordance with the results of other studies $(24,31)$. It has been shown that peripheral blood-derived $\mathrm{CD}^{+}$and $\mathrm{CD}^{+} \mathrm{T}$ cells may mediate the cytotoxicity of human gliomas via a mechanism independent of TNF in vitro (31). The observation of $\mathrm{T}$ cells inside the necrosis area in the present study suggests its role in the induction of necrosis. However, OX8 showed neither a similar distribution nor morphology to the detected $\mathrm{W} 3 / 13^{+}$cells. This may be attributed to the time course of the innate and adaptive immune reaction. Further study is required to find the underling mechanism.

In summary, in the present study, the early accumulation of diverse subsets of activated macrophages/microglia, including reactive $\mathrm{ED}^{+}$and $\mathrm{EMAPII}^{+}$cells, was observed in rat C6 gliomas. Notably, the $\mathrm{CD}^{+}$cells shared a similar distribution and morphology with these two subpopulations rather than the $\mathrm{W} 3 / 13^{+} \mathrm{T}$ cells. The abundance of this subpopulation and their strategic location highlights the possible comprehensive roles of these cells in glioma development, and the orchestration of active macrophages could be a potential research area for studying the development of immunotherapeutic antitumor agents in glioma.

\section{Acknowledgements}

The authors would like to thank Professor H. J. Schluesener for providing the C6 glioma rat brain tissue library and for assistance with immunostaining. This study was supported by the National Nature Science Foundation of China (grant no. 31170851).

\section{References}

1. Jovčevska I, Kočevar N and Komel R: Glioma and glioblastomahow much do we (not) know? Mol Clin Oncol 1: 935-941, 2013.

2. Platten M, Ochs K, Lemke D, Opitz C and Wick W: Microenvironmental clues for glioma immunotherapy. Curr Neurol Neurosci Rep 14: 440, 2014.

3. Glass R and Synowitz M: CNS macrophages and peripheral myeloid cells in brain tumours. Acta Neuropathol 128: 347-362, 2014.

4. Jackson CM, Lim M and Drake CG: Immunotherapy for Brain Cancer: Recent progress and future promise. Clin Cancer Res 20: 3651-3659, 2014.

5. Sloan AE, Dansey R, Zamorano L, Barger G, Hamm C, Diaz F, Baynes R and Wood G: Adoptive immunotherapy in patients with recurrent malignant glioma: Preliminary results of using autologous whole-tumor vaccine plus granulocyte-macrophage colony-stimulating factor and adoptive transfer of anti-CD3-activated lymphocytes. Neurosurg Focus 9: e9, 2000.

6. Atkinson LL, Merchant RE, Ghatak NR and Young HF: Sterile abscesses in glioma patients treated by intraparenchymal injection of lymphokine-activated killer cells and recombinant interleukin-2: Case reports. Neurosurgery 25: 805-810, 1989.

7. Badie B and Schartner J: Role of microglia in glioma biology. Microsc Res Tech 54: 106-113, 2001.

8. Lerch JK, Puga DA, Bloom O and Popovich PG: Glucocorticoids and macrophage migration inhibitory factor (MIF) are neuroendocrine modulators of inflammation and neuropathic pain after spinal cord injury. Semin Immunol 26: 409-414, 2014.

9. Doens D and Fernández PL: Microglia receptors and their implications in the response to amyloid $\beta$ for Alzheimer's disease pathogenesis. J Neuroinflammation 11: 48, 2014.

10. Onore CE, Schwartzer JJ, Careaga M, Berman RF and Ashwood P: Maternal immune activation leads to activated inflammatory macrophages in offspring. Brain Behav Immun 38: 220-226, 2014.

11. Zhou W and Bao S: Reciprocal supportive interplay between glioblastoma and tumor-associated macrophages. Cancers (Basel) 6: 723-740, 2014.

12. Streit WJ: Microglia as neuroprotective, immunocompetent cells of the CNS. Glia 40: 133-139, 2002.

13. Kempermann G and Neumann H: Neuroscience. Microglia: The enemy within? Science 302: 1689-1690, 2003.

14. Rock RB, Gekker G, Hu S, Sheng WS, Cheeran M, Lokensgard JR and Peterson PK: Role of microglia in central nervous system infections. Clin Microbiol Rev 17: 942-964, 2004. 
15. Ladeby R, Wirenfeldt M, Garcia-Ovejero D, Fenger C, Dissing-Olesen L, Dalmau I and Finsen B: Microglial cell population dynamics in the injured adult central nervous system. Brain Res Brain Res Rev 48: 196-206, 2005.

16. Mueller CA, Schluesener HJ, Conrad S, Meyermann R and Schwab JM: Lesional expression of a proinflammatory and antiangiogenic cytokine EMAP II confined to endothelium and microglia/macrophages during secondary damage following experimental traumatic brain injury. J Neuroimmunol 135: 1-9, 2003.

17. Brabeck C, Michetti F, Geloso MC, Corvino V, Goezalan F, Meyermann R and Schluesener HJ: Expression of EMAP-II by activated monocytes/microglial cells in different regions of the rat hippocampus after trimethyltin-induced brain damage. Exp Neurol 177: 341-346, 2002.

18. Fang KM, Wang YL, Huang MC, Sun SH, Cheng H and Tzeng SF: Expression of macrophage inflammatory protein-1 $\alpha$ and monocyte chemoattractant protein-1 in glioma-infiltrating microglia: Involvement of ATP and $\mathrm{P}_{2} \mathrm{X}_{7}$ receptor. J Neurosci Res 89: 199-211, 2011.

19. Gupta S: Role of dendritic cells in innate and adaptive immune response in human aging. Exp Gerontol 54: 47-52, 2014.

20. Popovich PG, van Rooijen N, Hickey WF, Preidis G and McGaughy V: Hematogenous macrophages express CD8 and distribute to regions of lesion cavitation after spinal cord injury. Exp Neurol 182: 275-287, 2003.

21. Zhang Z, Artelt M, Burnet M, Trautmann K and Schluesener HJ Early infiltration of $\mathrm{CD}^{+}$macrophages/microglia to lesions of rat traumatic brain injury. Neuroscience 141: 637-644, 2006.

22. Grobben B, De Deyn PP and Slegers H: Rat C6 glioma as experimental model system for the study of glioblastoma growth and invasion. Cell Tissue Res 310: 257-270, 2002.

23. Damoiseaux JG, Döpp EA, Calame W, Chao D, MacPherson GG and Dijkstra CD: Rat macrophage lysosomal membrane antigen recognized by monoclonal antibody ED1. Immunology 83 : $140-147,1994$
24. Perrin G, Schnuriger V, Quiquerez AL, Saas P, Pannetier C, de Tribolet N, Tiercy JM, Aubry JP, Dietrich PY and Walker PR Astrocytoma infiltrating lymphocytes include major $\mathrm{T}$ cell clonal expansions confined to the CD8 subset. Int Immunol 11: 1337-1350, 1999.

25. Yamashiro S, Takeya M, Nishi T, Kuratsu J, Yoshimura T, Ushio Y and Takahashi K: Tumor-derived monocyte chemoattractant protein-1 induces intratumoral infiltration of monocyte-derived macrophage subpopulation in transplanted rat tumors. Am J Pathol 145: 856-867, 1994.

26. Schroeter M, Stoll G, Weissert R, Hartung HP, Lassmann H and Jander S: CD8+ phagocyte recruitment in rat experimental autoimmune encephalomyelitis: Association with inflammatory tissue destruction. Am J Pathol 163: 1517-1524, 2003.

27. Stoll G, Jander S and Myers RR: Degeneration and regeneration of the peripheral nervous system: From Augustus Waller's observations to neuroinflammation. J Peripher Nerv Syst 7: 13-27, 2002.

28. Weissenböck H, Hornig M, Hickey WF and Lipkin WI Microglial activation and neuronal apoptosis in Bornavirus infected neonatal Lewis rats. Brain Pathol 10: 260-272, 2000.

29. Marc T: Brain development and the immune system: An introduction to inflammatory and infectious diseases of the child's brain. Handb Clin Neurol 112: 1087-1089, 2013.

30. Hirji N, Lin TJ, Bissonnette E, Belosevic M and Befus AD Mechanisms of macrophage stimulation through CD8: Macrophage CD8alpha and CD8beta induce nitric oxide production and associated killing of the parasite Leishmania major. J Immunol 160: 6004-6011, 1998

31. Baltuch GH, Villemure JG, McCrea E and Antel JP: $\mathrm{T}$ cell-mediated cytotoxicity of human gliomas: A tumor necrosis factor-independent mechanism. Neurosurgery 35: 450-456, 1994 